\title{
Avances de la inteligencia artificial en salud
}

\section{Advances in artificial intelligence in health}

\section{Avanços na inteligência artificial em saúde}

\author{
Diana Nancy Martínez-García ${ }^{\text {I }}$ \\ dnmartinezg@outlook.com \\ Violeta Maricela Dalgo-Flores II \\ violeta.dalgo@espoch.edu.ec \\ José Luis Herrera-López III \\ j1.herrera@uta.edu.ec \\ Eulalia Isabel Analuisa-Jiménez IV \\ ei.analuisa@uta.edu.ec \\ Evelin Fernanda Velasco-Acurio $\mathrm{V}$ \\ ef.velasco@uta.edu.ec
}

*Recibido: 17 de mayo de 2019 *Aceptado: 15 de junio de 2019 * Publicado: 05 de julio de 2019

\footnotetext{
${ }^{\text {I }}$ Magíster en Gerencia Informática, Magíster en Tecnologías para la Gestión y Práctica, Ingeniera en Sistemas Computacionales, Independiente, Universidad Católica del Ecuador, Quito, Ecuador.

II Magíster Universitario en Técnicas Experimentales en Química, Ingeniera Bioquímica, Docente de la Escuela Superior Politécnica de Chimborazo, (ESPOCH), Riobamba, Ecuador.

III Magíster en Enfermería Quirúrgica, Licenciado en Enfermería, Docente de la Universidad Técnica de Ambato, Ambato, Ecuador.

IV Magíster en Enfermería Quirúrgica, Licenciada en Enfermería, Docente de la Universidad Técnica de Ambato, Ambato, Ecuador.

${ }^{v}$ Magíster en Enfermería Quirúrgica, Licenciada en Enfermería, Docente de la Universidad Técnica de Ambato, Ambato, Ecuador.
} 


\title{
Resumen
}

La inteligencia artificial (IA) tiene el potencial de traer beneficios sin precedentes para la humanidad, por lo tanto, vale la pena investigar cómo maximizar estos beneficios, mientras que evita peligros potenciales. Este artículo de revisión da numerosos ejemplos de este valor, al tiempo que se realiza un análisis del avance en los últimos años y su aprovechamiento en diferentes áreas. Se evidencia el éxito en la búsqueda de inteligencia artificial y los beneficios sin precedentes a la humanidad, al tiempo que las investigaciones sobre IA deben asegurar que el impacto futuro sea beneficioso.

Palabras clave: Inteligencia artificial; tecnología biomédica; informática aplicada a la enfermería; tecnología de la información.

\begin{abstract}
Artificial intelligence (AI) has the potential to bring unprecedented benefits to humanity, therefore, it is worth investigating how to maximize these benefits, while avoiding potential dangers. This review article gives numerous examples of this value, while an analysis of progress in recent years and its use in different areas is made. There is evidence of success in the search for artificial intelligence and the unprecedented benefits to humanity, while research on AI must ensure that the future impact is beneficial.
\end{abstract}

Key words: Artificial intelligence; biomedical technology; nursing informatics; information technology.

\section{Resumo}

A inteligência artificial (IA) tem o potencial de trazer benefícios sem precedentes para a humanidade, portanto, vale a pena investigar como maximizar esses benefícios, evitando perigos potenciais. Este artigo de revisão fornece numerosos exemplos desse valor, enquanto uma análise é feita do progresso feito nos últimos anos e seu uso em diferentes áreas. O sucesso na busca por inteligência artificial e os benefícios sem precedentes para a humanidade são evidenciados, enquanto a pesquisa em IA deve garantir que o impacto futuro seja benéfico. 
Palavras-chave: Inteligência artificial; tecnologia biomédica; informática aplicada à enfermagem; tecnologia da informação.

\section{Introducción}

La inteligencia artificial (IA) comprende la simulación de procesos de inteligencia humana por parte de las máquinas mediante la interacción con sistemas informáticos (1). Dentro de estos procesos se consideran la adquisición de gran cantidad de información y reglas que ayuden a procesar la misma, para realizar las acciones solicitadas, el razonamiento mediante el uso de regulaciones con el objetivo de que el proceso de decisión le permita llegar a conclusiones aproximadas o definitivas y también la autocorrección, que permita realizar correcciones a los procesos y mediante éste se logre un aprendizaje retroactivo (2).

Según las principales tendencias en el ciclo Hart de Gartner para tecnologías emergentes (Top Trends in the Gartner Hype Cycle for Emerging Technologies, 2017) indica que para los próximos 10 años la IA, se convertirá en la tecnología de mayor impacto debido a diversos factores como la gran cantidad de datos e información, un gran poder computacional y al avance en redes neurales profundas. En un contexto propio de la aplicación de tecnologías en el área de salud, aún queda mucho por conocer y mucho más por hacer, pero es imprescindible empezar por reconocer la importancia de las inclusión de este tipo de desarrollos tecnológicos en este ámbito específico, resaltar las ventajas y ver en las desventajas que puedan presentarse retos que impliquen redoblar esfuerzos para obtener el mejor provecho para el beneficio de la comunidad $(3,4)$.

El término Inteligencia Artificial (Artificial Intelligence. AI) se le atribuye a John McCarthy, informático norteamericano que, en el año de 1956 durante una conferencia en Dartmouth, acuño el término, haciendo referencia a la conjetura de que algún día se podría proporcionar información tan precisa a mecanismos o dispositivos electrónicos que existiría la posibilidad de emular el pensamiento y libre albedrío humano (5).

En la actualidad, inteligencia artificial es un término general de gran alcance, que abarca desde la automatización de procesos robóticos hasta la robótica actual. Ha alcanzado relevancia básicamente en función de grandes volúmenes de datos e información, el incremento en la velocidad y capacidad de proceso de los equipos informáticos, lo asequible de las nuevas tecnologías, la difusión y 
propagación de las conexiones a internet, entre otros aspectos, que han permitido dar a conocer los avances generados en diferentes ámbitos, lo que ha despertado el interés de la sociedad por incursionar en este tipo de soluciones (6).

Más de medio siglo después, aún no existen ordenadores que emulen el pensamiento y libre albedrío humano. Las soluciones que existen en el mercado y otras tecnologías que actualmente continúan en desarrollo se mueven, hablan y responden, pero considerar que los avances de inteligencia artificial consigan, en estos momentos, equiparar a la vida, aún es un precepto que se visualiza lejano (7). En el año de 1978 McCarthy ya temía esto, y expresó que para desarrollar una IA en todo su potencial se requeriría del trabajo de "1,7 Einsteins, 2 Maxwells, 5 Faradays y la financiación de 0,3 proyectos Manhattan, siempre y cuando ese proyecto viniera después de los necesarios descubrimientos conceptuales" (8).

Los diversos estudios y aplicaciones permiten determinar diferentes tipos de inteligencia artificial mismos que se pueden ejemplificar de la siguiente manera:

Los sistemas de IA débil. Conocida también como IA estrecha, está diseñada y adiestrada para tareas particulares. Los recientemente incorporados asistentes personales virtuales, como Siri de Apple ${ }^{\circledR}$ o Alexa de Amazon ${ }^{\circledR}$, son una forma de débil de AI.

La IA fuerte, conocida también como inteligencia general artificial, presenta habilidades cognitivas humanas generalizadas, lo que le permite encontrar soluciones cuando se le presenta tareas desconocidas. Alan Turing, matemático considerado padre de la computación, desarrolló en 1950 un método utilizado para establecer si una computadora realmente puede desarrollar competencias cognitivas y pensar como un humano (9).

Arend Hintze, profesor asistente de biología integradora e ingeniería y ciencias de computación en la Universidad Estatal de Michigan, clasifica la inteligencia artificial en cuatro tipos. Las Máquinas reactivas, que son computadoras o robots que tienen la capacidad de reaccionar a situaciones determinadas, carecen de la capacidad de almacenar recuerdos para utilizarlos como experiencias para la toma de decisiones. Las máquinas de memoria limitada tipo II, tienen memoria limitada y la capacidad para utilizar experiencias que les sirvan en la toma de decisiones; sin embargo, estos recuerdos no son perennes, tienen una duración de corto plazo lo que impide que recuerde decisiones pasadas (10). 
La teoría de la mente en máquinas tipo III, representan una bifurcación entre las máquinas que actualmente se están construyendo y aquellas que se construirán en el futuro; éstas tendrán la capacidad de "formar representaciones sobre el mundo, pero también sobre otros agentes o entidades en el mundo" (11). En psicología, esto se llama teoría de la mente: "la comprensión de que las personas, criaturas y objetos en el mundo, pueden tener pensamientos y emociones que afectan su propio comportamiento". Las máquinas de autoconciencia tipo IV, tratan de emular lo más posible los aspectos de la inteligencia humana, ya que expanden la teoría de la mente, son conscientes de ellas mismas y comprenden el concepto de un individuo en relación con los demás.

El objetivo de la presente revisión bibliográfica fue fundamentar teóricamente los avances de las tecnologías basadas en inteligencia artificial aplicadas al área de salud.

\section{Desarrollo}

La inclusión de tecnologías en diferentes ámbitos de la vida cotidiana ha demarcado un antes y un después en la manera en cómo se desarrolla esa cotidianeidad. Actualmente no es posible pensar en un mundo evolucionado sin que el desarrollo tecnológico haya formado parte relevante de ese desarrollo (13), muchas formas de automatización de procesos han permitido que estos se realicen con mayor agilidad y sin duda, las soluciones informáticas están involucradas directamente en estas nuevas capacidades que la vida evolucionada requiere para desenvolverse con normalidad (14). El uso de nuevas tecnologías es un hito fundamental en el desarrollo de la historia humana en todas sus etapas, es posible evidenciar avances que contribuyen al mejoramiento de la calidad de vida de las personas y entre éstos se encuentran, justamente, los avances en IA aplicados a la salud (15).

La IA es una de las tecnologías que ha captado la atención a nivel mundial, por la prestaciones que promete, aunque ésta sigue desarrollándose y perfeccionándose constantemente, en la actualidad es posible ser testigo de los avances que ofrece (16), se le considera como una de las tecnologías más prominentes de los últimos tiempos y genera muchas expectativas, ya que conjuntamente con la mejora de la capacidad de proceso de los computadores, permite mayor rapidez en el procesamiento de información, lo que se traduce en consecución de objetivos y obtención de resultados que pone a prueba la validez de las teorías planteadas, hace más de 50 años por quién puso su esfuerzo y contribuyó a la historia de la humanidad al plantear paradigmas que despertaran el interés por 
concebir ideas, para algunos sueños absurdos, de que las máquinas puedan dar otros servicios, más allá, que únicamente cálculos y que incluso adquieran la capacidad de aprender (17).

A este se refiere la IA, a la capacidad que pudieran tener las máquinas para aprender por ellas mismas, emulando lo más posible a los procesos de inteligencia humana (18). Este concepto, que suena como ciencia ficción, se basa en reglas y métodos lógicos de aprendizaje. Las máquinas no pueden pensar por sí solas, al menos no aún, pero si se está trabajando en darle la capacidad de aprender, desaprender y reaprender situaciones cotidianas, lo que brinda un panorama general de interacción para tomar decisiones, esto lo hacen a través de procesos, como adquisición de información, uso de dicha información mediante reglas de utilización específicas, que da como resultado un proceso de razonamiento que mediante el uso de normas y reglas permita a las máquinas llegar a conclusiones definitivas o aproximadas y mediante la autocorrección adquirirán la posibilidad de rehacer sus decisiones para obtener resultados esperados (19). Sin embargo, esta tecnología también ha encontrado detractores en aquellos que consideran, que darles a las máquinas la capacidad de tomar decisiones por ellas mismas, conllevaría una debacle de la humanidad y en casos más extremos la aniquilación del mundo tal y como lo conocemos (20).

Estas ideas, fantasiosas en muchos casos, han sido plasmadas en películas futuristas, en dónde la influencia de la industria cinematográfica ha causado gran impacto en la idea que las personas se hacen respecto a las máquinas inteligentes, robots de servicios, etc. (21). Muchos científicos, desarrolladores de tecnologías y políticos, se han sumado a este temor, hasta el momento infundado, como el Dr. Stephen Hawking, quien en el año 2014, en una entrevista mencionó que "la inteligencia artificial podría resultar en el fin de la raza humana" (22), a pesar de los pronósticos desalentadores que personalidades relevantes del mundo científico puedan considerar, el desarrollo de las tecnologías, beneficios y desventajas, está supeditado al uso que los seres humanos hagan de ella (23). Los investigadores que defienden las posturas del desarrollo de la IA al servicio de la humanidad, sostienen que es poco probable que una IA súper inteligente exhiba emociones humanas y por ende que ésta se vuelva intencionalmente malvada $(24,25)$.

A pesar del contexto que envuelve el desarrollo en IA, es innegable el vertiginoso crecimiento que ha conseguido en los últimos 5 años. Las IA aplicadas a la salud podrían ahorrar 150.000 millones de 
dólares a la industria médica. Además, el mercado tiene mucho potencial de crecimiento. En 2014 era de 600 millones de dólares, y en 2020 se estima que será de 6.600 millones, 11 veces (25).

La inteligencia artificial ha sido categorizada en base a sus prestaciones y capacidades, de aquí tenemos que las máquinas reactivas son aquellas que responden a situaciones específicas en tareas únicas un ejemplo de esto es Deep Blue, que es un programa de ajedrez de IBM que venció a Garry Kasparov, campeón del mundo en ajedrez en los años noventa. Deep Blue identificaba piezas en el tablero de ajedrez y realizaba predicciones, pero al no tener memoria y no era capaz de usar experiencias pasadas para informar a las futuras. Analizaba movimientos posibles -propios y los de sus oponentes- y elegía el movimiento más estratégico (26).

Las máquinas de memoria limitada pueden usar experiencias pasadas para informar decisiones futuras. Algunas de las funciones de toma de decisiones en vehículos autónomos han sido diseñadas de esta manera. Las observaciones son utilizadas para informar las acciones que ocurren en un futuro no tan lejano, como un coche que ha cambiado de carril. Estas observaciones no se almacenan permanentemente. Teoría de la mente es un término psicológico que hace referencia a la comprensión de que los demás tienen sus propias creencias, deseos e intenciones que afectan las decisiones que toman. Este paradigma dentro de la AI aún no existe. Autoconocimiento es una categoría, donde los sistemas de AI tienen un sentido de sí mismos, tienen conciencia. Las máquinas con conciencia de sí comprenden su estado actual y pueden usar la información para inferir lo que otros están sintiendo $(27,28)$.

De las aplicaciones más populares de IA, las utilizadas en el sector salud son las que representan la mayor proporción, en cuanto al uso y expectativas refieren; éstas son asistente robótico para cirugías o robot-assisted surgery, este asistente da la posibilidad al profesional médico, realizar operaciones sin la necesidad de que doctor y paciente se encuentren en el mismo espacio geográfico, las virtual nursing assistant o enfermeras virtuales son aplicaciones de IA que asisten al paciente en cualquier momento que este desee, mediante una aplicación que puede descargarse de internet hacia un dispositivo móvil, la enfermera virtual puede hacer seguimiento diario de los valores y mediciones que el paciente mismo puede realizarse desde su casa y todo con la guía profesional virtual que incluso 


\section{Avances de la inteligencia artificial en salud}

tiene la capacidad de que en función de la información que el usuario provea recomendar tratamientos o derivar a un centro de salud si así lo considera pertinente (29-32).

Uno de los mejores ejemplos de la aplicación de IA en salud es Watson. El superordenador de IBM que ya es capaz de dar diagnósticos de cáncer con una precisión del 83\%, y sugiere posibles tratamientos al profesional médico. Watson también puede realizar un seguimiento individualizado de cada paciente a nivel genético. E incluso ya está identificando medicamentos nuevos y la relación entre medicamentos existentes. DeepMind, la división de investigación de inteligencia artificial de Google, se ha aliado con el servicio nacional de salud británico. El objetivo de la compañía de Alphabet es crear una aplicación, Streams, que centraliza la información sobre un paciente. La gracia es que Streams puede generar alertas en base a esa información, permitiendo al profesional sanitario actuar con rapidez. Sentrian apunta aún más allá, el objetivo es prever cuándo una persona se puede enfermar. Así podrá tratarse incluso antes de que tenga que ir al hospital, reduciendo las asistencias a los centros hospitalarios. Para ello usa biosensores, y machine learning para analizar datos (28).

\section{Conclusión}

La IA en el campo de la salud, plantea el desarrollo de nuevas herramientas informáticas para el descubrimiento de fármacos y dianas moleculares, utilizando técnicas de ingeniería informática e inteligencia artificial. En consecuencia, la información estructural de las moléculas se codificó en los índices topológicos de los grafos moleculares, con la ayuda de nuevos programas informáticos específicos implementados. La inclusión de nuevas tecnologías es distintos ámbitos de la vida es inminente, sin embargo, no es posible predecir las consecuencias de su uso en un futuro, más bien lo que el usuario común puede hacer es albergar la esperanza de que las mismas mejoren las condiciones de vida del ser humano y no se conviertan en la amenaza que actualmente varios vaticinan.

\section{Referencias Bibliográficas}

1. Barber, F. 10 años de "Inteligencia Artificial", Revista Iberoamericana de I.A. Inteligencia Artificial. Revista Iberoamericana de Inteligencia Artificial [Internet]. 2007;11(33):iii-vi.

2. Bostrom N. The Superintelligent Will: Motivation and Instrumental Rationality in Advanced Artificial Agents. Minds and Machines 2012; 22(2): 71-85. dx.doi.org/10.1007/ s11023-0129281-3 
3. Brynjolfsson E, McAfee A. The Second Machine Age: Work, Progress, and Prosperity in a Time of Brilliant Technologies. 2014. New York: W.W. Norton \& Company.

4. Brynjolfsson E, McAfee A, Spence M. Labor, Capital, and Ideas in the Power Law Economy. Foreign Affairs 2014; 93(4): 44.

5. Tegmark M. Friendly Artificial Intelligence: The Physics Challenge. In Artificial Intelligence and Ethics, ed. T. Walsh, AAAI Technical Report WS-15-02, 2015. p87-89. Palo Alto, CA: AAAI Press.

6. Vladeck, D. C. 2014. Machines Without Principals: Liability Rules and Artificial Intelligence. Washington Law Review 89(1): 117.

7. Chu W, Ghahramani Z. Preference Learning with Gaussian Processes. In Proceedings of the 22nd International Conference on Machine Learning, 2005. 137-144. New York: Association for Computing Machinery. dx.doi.org/10.1145/ 1102351.1102369

8. Asaro, P. M. 2006. What Should We Want from a Robot Ethic? International Review of Information Ethics 6(12): 9-16.

9. Top Trends in the Gartner Hype Cycle for Emerging Technologies, 2018. Smarter With Gartner. 2018. Disponible en: https:/www.gartner.com/smarterwithgartner/top-trends-in-the-gartnerhype-cycle-for-emerging-technologies-2017/

10. Lahoz-Beltra R. Bioinformática: Simulación, vida artificial e inteligencia artificial. Ediciones Díaz de Santos; España. 2010. 610 p.

11. González-Díaz H, Munteanu C, Postelnicu L, Prado-Prado F, Gestal M, Pazos A. LIBPPred: web server for lipid binding proteins using structural networkparameters; PDB mining of human cancer biomarkers and drug targets in parasites and bacteria. Molecular BioSystems 2012; 8: 851-862.

12. Akaike M, Fukutomi M, Nagamune M. Simulation-based medical education in clinical skills laboratory. J Med Invest 2012;59:28-35

13. Dávila-Cervantes A. Simulación en Educación Médica. Investigación en Educación Médica, 2014; vol. 3 (10): 100-105

14. Gallardo E, Carmen M del, Ávila Ávila R. Aplicaciones de la inteligencia artificial en la Medicina: perspectivas y problemas. ACIMED. mayo de 2008;17(5):10-19.

15. Mayer M, Leis Á. Concepto y aplicaciones de la Web 3.0: una introducción para médicos. Aten 
Primaria. 2010;42(5):292-296.

16. Serrano P. Inteligencia artificial crucial para 4 de cada 10 empresa. 2019. Disponible en: https://www.nobbot.com/general/inteligencia-artificial-2020/

17. Taraborrelli D, Gala R. Génesis y actualidad de la inteligencia artificial (IA) en las instituciones públicas de la Argentina, una mirada desde los ESCyT. 2015. XI Jornadas de Sociología. Facultad de Ciencias Sociales, Universidad de Buenos Aires, Buenos Aires. Dirección estable: https://www.aacademica.org/000-061/366 Haugeland J. La inteligencia artificial. Siglo XXI; 1988. $260 \mathrm{p}$.

18. González Polanco L, Pérez Betancourt G. La minería de datos espaciales y su aplicación en los estudios de salud y epidemiología. Revista Cubana de Información en Ciencias de la Salud, 2013; 24(4), 482-489.

19. Correa A. Neurociencia aplicada: el cerebro al servicio de la humanidad. Ciencia Cognitiva: Revista Electrónica de Divulgación, 2008; 2:1, 38-40

20. Jensen R, Lopes MHB de M. Nursing and fuzzy logic: an integrative review. Rev Lat Am Enfermagem. 2011;19(1):195-202.

21. PAHO. Publication: Modelos y teorías en enfermería, 5a. ed. - Paltex. [citado 20. de mayo de 2019]. Disponible en: http://paltex.paho.org/Publication/Index?publicationId=373

22. García MR, Rodríguez J. Sistemas basados en el conocimiento. Rev Vínculos. 2004;1(1):37-44.

23. González AT. Software de aplicación de técnicas de aprendizaje automático para el diagnóstico médico. https://www.researchgate.net/publication/283463066_SOFTWARE_DE_APLICACION_DE_T ECNICAS_DE_APRENDIZAJE_AUTOMATICO_PARA_EL_DIAGNOSTICO_MEDICO/lin $\mathrm{k} / 56391 \mathrm{aeb} 08 \mathrm{aecf1d} 92 \mathrm{a} 9 \mathrm{bdc} 3 / 11$.

24. Munteanu CR. Técnicas de ingeniería informática e inteligencia artificial para clasificación : aplicaciones para el descubrimiento de fármacos y dianas moleculares. 2013 [citado 22 de mayo de 2018]; Disponible en: http://ruc.udc.es/dspace/handle/2183/10308

25. Dávila Hernández F, Sánchez Corales Y. Técnicas de minería de datos aplicadas al diagnóstico de entidades clínicas. Rev Cuba Informática Médica. 2012;4(2):174-83.

26. Alcolea G, Esteban E. Vida e inteligencia artificial. ACIMED. 2009;19(1):0-0. 
27. Stuart Russell S, Dewey D, Tegmark M. Research Priorities for Robust and Beneficial Artificial Intelligence. AI Magazine, 2015; Vol 36 No 4: 105-114. Doi: https://doi.org/10.1609/aimag.v36i4.2577

28. Okuda Y, Quinones J. The use of simulation in the education of emergency care providers for cardiac emergencies. Int J Emerg Med 2008;1(2):73-77.

29. Barsuk JH, Cohen ER, Feinglass J. Use of simulation-based education to reduce catheter-related bloodstream infections. Arch Intern Med 2009;169(15):1420-1423.

30. McGaghie WC, Issenberg SB, Cohen ER. Does simulationbased medical education with deliberate practice yield better results than traditional clinical education? A meta-analytic comparative review of the evidence. Acad Med 2011;86(6):706-711.

31. Sroka G, Feldman LS, Vassiliou MC. Fundamentals of laparoscopic surgery simulator training to proficiency improves laparoscopic performance in the operating room-a randomized controlled trial. Am J Surg 2010;199:115-120.

32. Motola I, Devine LA, Chung HS. Simulation in health care education: a best evidence practical guide. AMEE Guide No. 82. Med Teach 2013;35(10):e1511-1530. 